\title{
Psicooncología
}

ISSN: 1696-7240

\section{La intervención psicológica en mindfulness con pacientes supervivientes de cáncer de mama. Revisión sistemática}

\author{
Raquel Calero'; Juan Antonio Cruzado ${ }^{2}$
}

Recibido: 30 de diciembre 2017 / Aceptado: 10 de febrero de 2018

Resumen. Objetivo. Valorar la eficacia de las intervenciones psicológicas en mindfulness con pacientes supervivientes de cáncer de mama. Método. Buscamos en las bases de datos: Pubmed, CINAHL y Psycinfo, investigaciones con intervenciones en mindfulness a pacientes adultas supervivientes de cáncer de mama, que incluyeran grupos experimentales y control, así como seguimiento tras finalizar las intervenciones durante el período de enero 2011 hasta octubre de 2017. Resultados. Se obtuvieron 10 investigaciones con diseños aleatorios controlados y seguimientos que aplicaron la intervención psicológica en mindfulness con supervivientes adultas de cáncer de mama. Conclusiones. Las intervenciones psicológicas en mindfulness inciden positivamente en la reducción de sintomatología psicológica y física de las pacientes supervivientes con cáncer de mama mejorando la calidad de vida de estas.

Palabras Claves: Cáncer de mama; supervivencia; mindfulness; calidad de vida.

\section{[en] The psychological intervention in mindfulness with surviving patients of breast cancer. Systematic review}

\begin{abstract}
Objetive. To assess the effectiveness of psychological interventions in mindfulness with patients surviving breast cancer. Method. We searched the databases: Pubmed, CINAHL and Psycinfo, investigations with interventions in mindfulness to adult patients surviving breast cancer, which included experimental groups and control and monitoring after completing the interventions during the period from January 2011 to October 2017. Results. We obtained 10 investigations with randomized controlled designs and follow-ups that apply the psychological intervention in mindfulness with adult breast cancer survivors. Conclusions. The psychological interventions in mindfulness positively affect the reduction of psychological and physical symptoms of the surviving patients with breast cancer improving their quality of life.
\end{abstract}

Keywords: Breast cancer; survivors; mindfulness; quality of life.

Sumario. 1. Introducción 2. Metodología 2.1. Estrategia de búsqueda, bases de datos, criterios de inclusión y exclusión 3. Resultados 4. Discusión 5. Conclusión 6. Referencias bibliográficas.

1 Raquel Calero. Universidad Complutense de Madrid. 28223- Madrid.

E-mail: racalero@ucm.es

2 Juan A. Cruzado. Universidad Complutense de Madrid. 28223-Madrid.

E-mail: jacruzad@ucm.es

* Dirección de correspondencia: Raquel Calero. Universidad Complutense de Madrid. 28223- Madrid.

E-mail: racalero@ucm.es 
Cómo citar: Calero R, Cruzado JA. La intervención psicológica en mindfulness con pacientes supervivientes de cáncer de mama. Revisión sistemática. Psicooncología 2018;15:75-88. Doi: 10.5209/ PSIC.59177.

\section{Introducción}

El cáncer de mama sigue siendo el tumor más diagnosticado entre las mujeres en España ${ }^{(1)}$ y Europa ${ }^{(2)}$. En el mundo es el más común y entre las mujeres es la neoplasia más frecuente, con un estimado de 1,67 millones de nuevos casos diagnosticados en $2012\left(25 \%\right.$ de todos los cánceres) ${ }^{(3)}$.

En España alrededor de 1.500.000 de personas han superado una enfermedad oncológica y las previsiones indican que se incrementarán un 50\% para el $2020^{(2)}$. Los últimos datos de los que disponemos en nuestro país indican que la supervivencia a 5 años será superior al $80 \%$ de los casos diagnosticados ${ }^{(4)}$. El diagnóstico precoz, el éxito de los tratamientos y el avance de los cribados están favoreciendo la supervivencia de un cada vez mayor número de pacientes.

Ser diagnosticada de cáncer de mama y superar los tratamientos implica múltiples necesidades de atención; además de problemas médicos, adaptaciones continuas a problemas sociales, mentales y emocionales, que limitan la calidad de vida de estas pacientes ${ }^{(5)}$. Así muchas de estas pacientes una vez superados los tratamientos presentaran secuelas físicas (cambios en la imagen corporal, fatiga, falta de energía, problemas en el sueño, etc.), neurocognitivas (disfunción cognitiva) y psicológicas (desorientación, ansiedad y depresión) ${ }^{(6,7)}$.

En los últimos años se ha incrementado la investigación en las intervenciones psicológicas destinadas a favorecer la calidad de vida en pacientes supervivientes de cáncer de mama. La naturaleza de las intervenciones son básicamente similares a las de las fases diagnósticas y de tratamiento en estas pacientes: psicoeducativas, terapias cognitivo conductuales, counselling grupal e individual y terapia de expresión emocional ${ }^{(8,9)}$. La intervención en psicoeducación, el apoyo emocional y social han encontrado escaso apoyo ${ }^{(10)}$. Las intervenciones cognitivo conductuales han mostrado ser eficaces a largo plazo en mejorar síntomas depresivos y calidad de vida en supervivientes de cáncer de mama ${ }^{(11)}$, y aunque la revisión de estas intervenciones ha encontrado beneficios a largo plazo en estas pacientes, los resultados no son concluyentes, por el número reducido de las muestras, la heterogeneidad y problemas metodológicos de los estudios ${ }^{(12)}$.

En los últimos años se han ido incluyendo terapias novedosas en la intervención psicológica con pacientes oncológicos que emplean el Mindfulness combinado con terapia cognitivo conductual. Su utilización en pacientes oncológicos está encaminada a reducir los niveles de estrés y los aspectos emocionales negativos ${ }^{(13)}$. Este tipo de intervenciones se han realizado con pacientes supervivientes ${ }^{(14)}$, demostrando que promueven el crecimiento postraumático. Shennan et al. ${ }^{(15)}$ hace una revisión de las intervenciones psicológicas basadas en Mindfulness empleadas con pacientes oncológicos diagnosticados de diferentes tumores y estadios de la enfermedad entre 2007 y 2011. Los resultados reflejan la existencia de una mejoría significativa en síntomas de ansiedad, depresión, estrés, dificultades sexuales, activación fisiológica y función inmune, o beneficios subjetivos en todas las 
intervenciones. Recomiendan continuar investigando los efectos de estas terapias a largo plazo y su utilidad y beneficio en pacientes oncológicos independientemente al tipo de tumor y estadio de la enfermedad donde se encuentre.

El Mindfulness empieza a utilizarse a finales de 1970 como intervención terapéutica destinada a reducir los síntomas de estrés y ansiedad y problemas con el sueño en poblaciones sanas en la Clínica de Reducción de Estrés de la Universidad de Massachusetts. Este programa, Mindfulness Based Stress Reduction (MBSR), consta de 8 sesiones semanales de 2,5 horas de duración. En ellas se practican; ejercicios de atención y relajación, meditación, yoga Hatha, así como explicaciones y diálogos sobre los procesos de la mente y el cuerpo. Las sesiones se realizan en grupos y alternan momentos de silencio con otros de exploración colectiva sobre estrategias para afrontar las situaciones complejas y difíciles de la vida, buscando además aplicaciones prácticas en el ámbito personal y profesional ${ }^{(16)}$.

A finales de 1990, aparecen modalidades de tratamiento que combinan Mindfulness con la terapia cognitivo-conductual (Mindfulness Based Cognitive Therapy, MBCT), ampliando su intervención a síntomas depresivos y el dolor crónico. Este programa diseñado para poblaciones clínicas en su origen se marca como objetivo prevenir y reducir las recaídas de pacientes que pasaron por un diagnóstico y tratamiento depresivo. El programa se desarrolla como el anterior a lo largo de 8 sesiones que incluyen herramientas que faciliten al paciente detectar un empeoramiento del estado anímico, habilidades de descentramiento y habilidades para ayudar a romper el vínculo afecto y pensamiento rumiativo que presentan estos pacientes. Tras años de aplicación sus creadores informaron que la aplicación de MBCT en trastorno de depresión o fibromialgia- resultaba eficaz ${ }^{(17)}$.

Por otro lado, el equipo que lidera Carlson ${ }^{(18)}$ a finales de 1990 también adaptó el programa MBSR a pacientes oncológicos e iniciaron investigaciones encaminadas a demostrar la validez y fiabilidad de estas intervenciones. Su programa: recuperación del cáncer basada en Mindfulness (Mindfulness Based Cancer Recovery, MBCR), fue desarrollado por su equipo y en la actualidad está destinado a personas que viven con cáncer. Incluyen 8 sesiones con un retiro de fin de semana y recomiendan una práctica diaria para casa como en el programa MBSR. El tiempo de las sesiones es más limitado del programa original de MBSR, con el fin de adaptarse a los síntomas (como el cansancio, falta de energía) que padecen los pacientes oncológicos. Los principios y prácticas centrales son esencialmente lo mismo e incluyen material específico para afrontar la sintomatología asociadas al cáncer; como son los problemas del sueño, el dolor y el miedo a la recurrencia del cáncer; y algunas estrategias de afrontamiento cognitivo basadas en principios de terapia cognitivo conductual. Utilizan además componentes de movimiento consciente a través de posturas de yoga y hacen énfasis en el proceso y apoyo grupal.

Otra versión de Mindfulness para afrontar la enfermedad oncológica es la de Bartley ${ }^{(19)}$, Terapia Cognitiva basada en Mindfulness para el cáncer (Mindfulnessbased Cognitive Therapy for Cancer, MBCT). Partiendo de los trabajos de Segal et al. ${ }^{(17)}$ y su experiencia clínica, adapta el MBCR a las necesidades de los pacientes oncológicos. En este manual para profesionales desarrolla un curso de Mindfulness de ocho semanas de duración similar al MBCT y dirigido especialmente a personas que padecen cáncer. El manual contiene un capítulo destinado a informar del 
seguimiento de más de 10 años de pacientes que participaron de la terapia, así como de los beneficios hallados en la calidad de vida de estos tras su aplicación.

La finalidad de este artículo es valorar la eficacia de las intervenciones psicológicas con Mindfulness en pacientes supervivientes de cáncer de mama. Las sintomatologías presentes en las pacientes supervivientes de cáncer de mama son similares a los síntomas con los que el MBSR, MBCT y MBCR muestran eficacia (estrés, ansiedad, problemas del sueño, depresión, dolor crónico). Sin embargo, se hace necesario llevar a cabo una revisión actualizada en la que se expongan en qué consisten estas intervenciones, la estructura de su programa, así como la valoración de su eficacia.

\section{Metodología}

\subsection{Estrategia de búsqueda, bases de datos, criterios de inclusión y exclusión}

Se llevó a cabo una revisión bibliográfica exhaustiva en las bases de datos de Pubmed, CINAHL y PsycINFO. En todas las bases de datos utilizamos la siguiente estrategia de búsqueda:

Descriptores: "Breast cancer AND survivor AND (depression OR anxiety OR anxious OR worried OR scared OR nervous OR cognitive OR quality of life OR fatigue OR pain OR body image OR lymphedema OR nausea OR vomiting OR sexual dysfunction OR sleep) AND mindfulness". Los términos de búsqueda fueron adaptados a cada base de datos e involucraban referencias cruzadas y combinadas de palabras clave.

En la selección de artículos tuvimos en cuenta como criterios de inclusión:

- Trabajos publicados desde enero de 2011 hasta el octubre de 2017.

- Pacientes mujeres con cáncer de mama en fase de supervivencia, estadio 0, I, II y III que no estuvieran recibiendo ni quimioterapia ni radioterapia, pudiendo estar tomando tratamiento hormonal.

- Intervenciones en pacientes adultas a partir de 18 años

- Investigaciones que incluían grupo control y seguimiento tras la intervención con el fin de garantizar la validez interna de las intervenciones.

Todas las publicaciones que no incluían estos requisitos quedaron excluidas de nuestro estudio.

\section{Resultados}

Se encontraron un total de 126 publicaciones en las bases de datos consultadas y tras eliminar las publicaciones duplicadas obtuvimos 73 artículos. Los resultados relativos al número y clasificación según características de las publicaciones encontrados en cada una de las bases de datos se exponen en la tabla 1.

Finalmente, se seleccionaron 10 artículos que cumplían los criterios de inclusión establecidos para esta revisión. Los 10 artículos seleccionados aparecen en PUBMED, 8 de ellos aparecen en CINHAL y 5 en PsycINFO.

En la tabla 2 aparece un resumen de los datos más destacados de cada investigación seleccionada. 


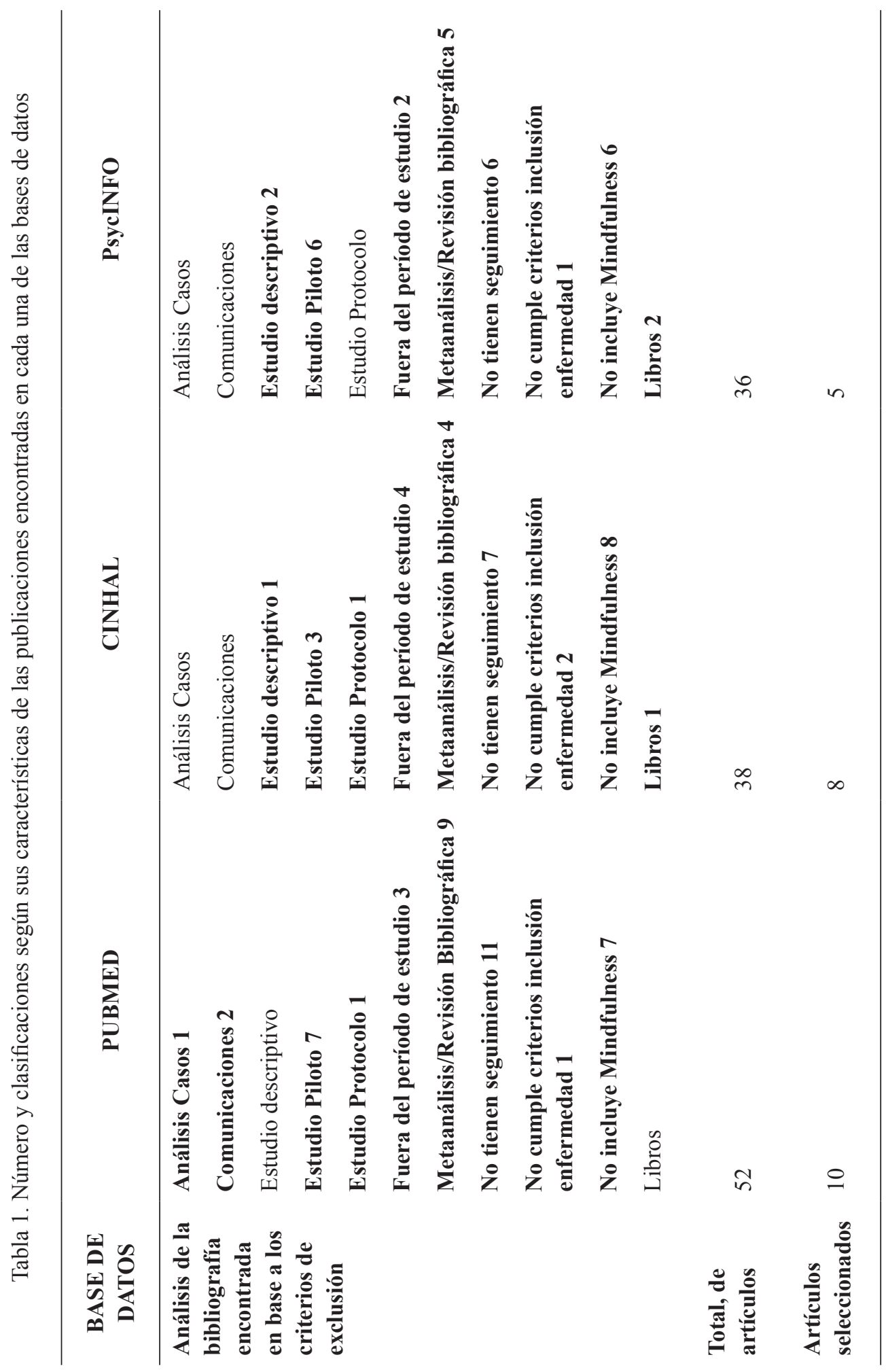




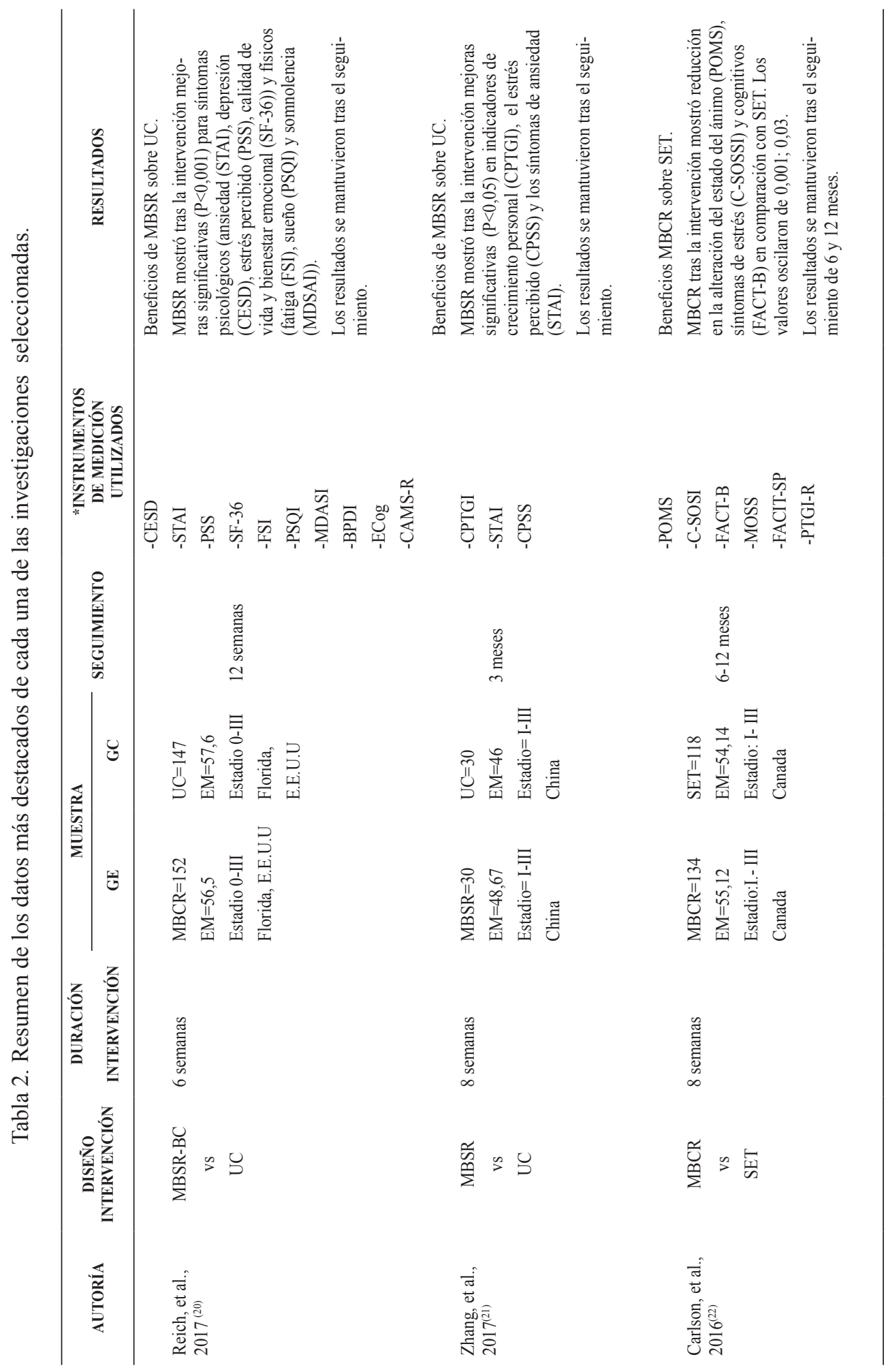




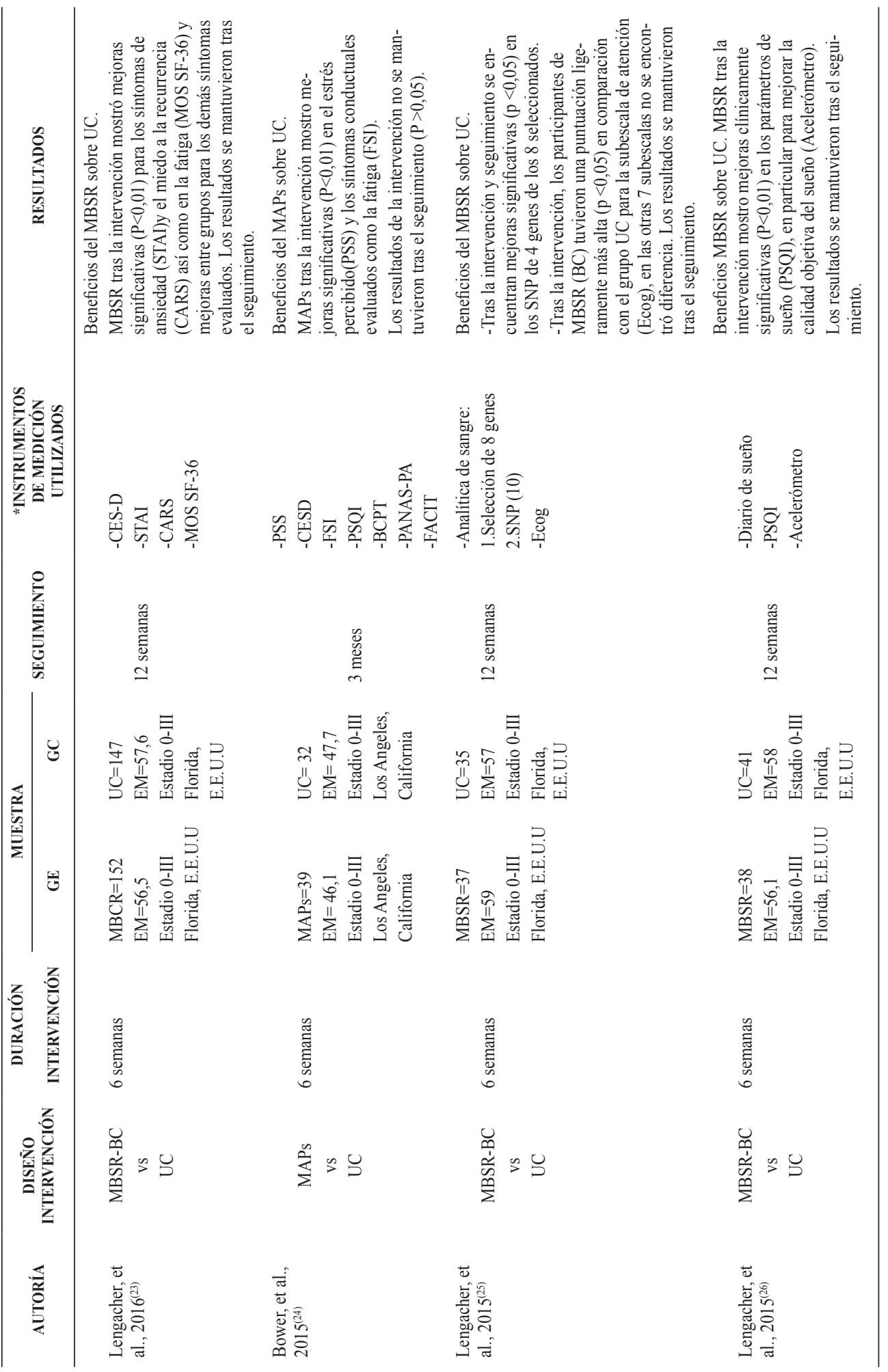




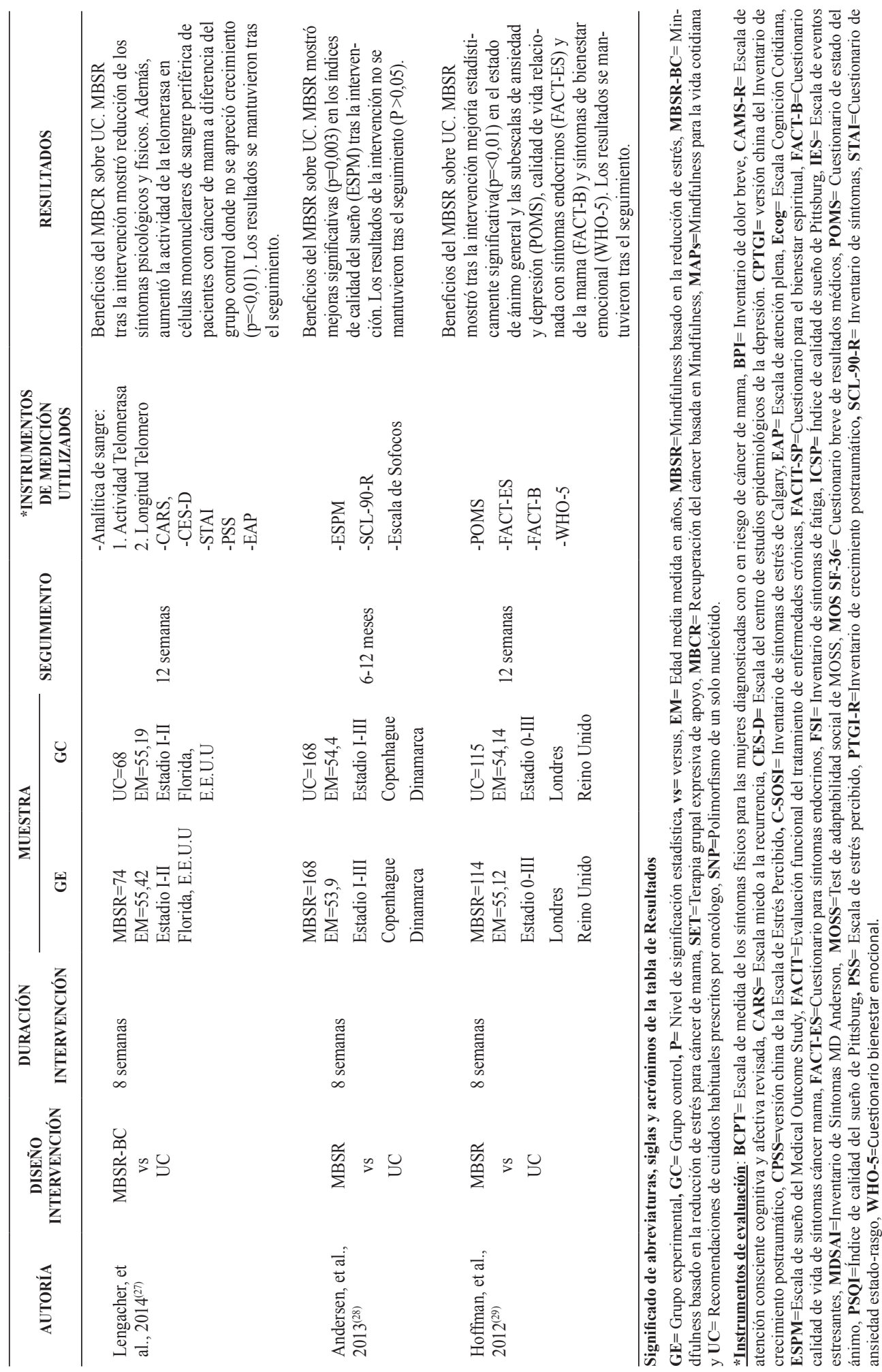


La tabla 2 resume los estudios que cumplían los criterios de elegibilidad. Se exponen los autores y el año de publicación, tipo de intervención y duración, muestra y dentro de ella la edad media de los grupos experimental y control, características del estadio de la enfermedad y país donde se realizó el estudio, duración del seguimiento, los instrumentos de medición utilizados y las conclusiones. Los estudios se organizan por año de publicación en orden descendente a partir de 2017. Dentro de un año determinado de publicación, los estudios se organizan alfabéticamente por el apellido del primer autor.

La mayoría de los estudios se realizaron en Estados Unidos $(n=5)^{(20,23,25-27)}$. La muestra cuenta con mujeres de origen americano, europeo y asiático y recoge un total de 1.839 mujeres supervivientes de cáncer de mama participantes que varían de 60 a 336 participantes por investigación. El rango de edad va de 18 a 75 años con una media total de edad de 55 años. Se incluyen exclusivamente mujeres supervivientes de cáncer de mama con estadios de 0-III. Todos los estudios se realizaron a estas pacientes en situación de no hospitalización y atendidas en fase de seguimiento en consultas ambulatorias.

De las 10 investigaciones encontradas, 9 emplean grupos controles (UC) que no reciben ningún tipo de intervención, pasan a listas de esperas y se les recomiendan que sigan los cuidados habituales prescritos por sus oncólogos. Una sola investigación ${ }^{(22)}$ compara la eficacia de la intervención en MBCR con la intervención en SET. Reich et al. ${ }^{(20)}$, Zhang et al. ${ }^{(21)}$, y el equipo liderado por Lengacher ${ }^{(23,25-27)}$ en todas sus investigaciones piden además a las pacientes del grupo UC que se abstengan de practicar meditación, técnicas de yoga y MBSR durante las inscripciones en el estudio.

Los seguimientos de 8 investigaciones se realizan a las 12 semanas de haber finalizado el programa de intervención y otras 2 incluyen los seguimientos más largos a los 6 y 12 meses del fin de la intervención ${ }^{(22,28)}$.

Con respecto al diseño de las intervenciones en mindfulness hemos encontrado diferencias en su estructuración. Las características de diferenciales son las siguientes:

- Dos investigaciones ${ }^{(28,29)}$ reproducen sin modificación el programa original de MBSR.

- Una investigación ${ }^{(21)}$ reproduce el programa original de MBSR a lo largo de 8 sesiones con 2 horas de duración por sesión, media hora menos que el original, adaptando el contenido a las necesidades de las pacientes supervivientes de cáncer de mama.

- Cinco investigaciones ${ }^{(20,23,25-27)}$ reproducen el programa original de MBSR adaptando el contenido y el tiempo de las sesiones a las necesidades de las pacientes supervivientes de cáncer de mama; preocupaciones y sintomatología. Imparten el programa en 6 semanas con 2 horas de duración por sesión.

- Una investigación ${ }^{(22)}$ sigue el programa de MBCR, similar en contenido y estructuración de tiempo al que emplean los investigadores anteriores, aunque incluyen temáticas de terapia cognitivo-conductual presentes en el $\mathrm{MBCT}^{(17)}$.

- Una investigación ${ }^{(24)}$ utiliza el programa: Mindfulness para la vida cotidiana (MAPs). Lo llaman intervención educativa y está basado en mindfulness, lo desarrollan en el Mindful Awareness Research Center (MARC), de la Universidad de California en Los Ángeles ${ }^{(30)}$. El programa se estructura en 6 sesiones grupales semanales de 2 horas de duración. Las sesiones incluyen la presentación de materiales teóricos sobre la atención plena, la relajación y 
la conexión mente-cuerpo; práctica experiencial de ejercicios de meditación y movimientos suaves (por ejemplo, caminar atento); y un componente psicoeducativo específico para supervivientes de cáncer. Los participantes son instruidos además como en MBSR y MBCR para practicar en casa las técnicas de atención plena diariamente, comenzando con 5 minutos por día y aumentando hasta 20 minutos progresivamente a lo largo del programa.

Los instrumentos de medición más usados son las escalas; CESD para evaluar depresión y PSS que evalúa el estrés percibido y el Cuestionario STAI para medir la ansiedad. Seguidas por el cuestionario de calidad de vida FACT-B, el POMS que evalúa estado anímico, y el miedo a la recaída CARS.

Las 10 investigaciones informan tras las intervenciones (MBSR, MBSR-BC, MBCR, MAPs $)$ de datos de mejora significativa $(\mathrm{p}<0,05)$ para síntomas psicológicos, físicos, cognitivos e inmunológicos (aumento de la telomerasa). 7 investigaciones sostienen además que tras el seguimiento los resultados se mantienen 12 semanas y 1 investigación encuentra mantenimiento de las mejoras en la reducción en la alteración del estado del ánimo, síntomas de estrés y cognitivos a los 6 y 12 meses tras haber finalizado la intervención.

\section{Discusión}

Una vez finalizadas los tratamientos las pacientes supervivientes de cáncer de mama presentan una elevada sintomatología psicológica y física, así como problemas cognitivos y las intervenciones en mindfulness llevan años de investigación informando sobre mejorías en la reducción de esta sintomatología potenciando la mejora en la reducción del estrés y la calidad de vida en general de estas pacientes tras las intervenciones ${ }^{(31-34)}$. No obstante, no podemos tomar estos resultados como concluyentes por la falta de rigor en el diseño de las investigaciones.

Nuestra revisión tenía por objetivo validar la eficacia de estas intervenciones de mindfulness en pacientes supervivientes de cáncer de mama, por este motivo en los criterios de inclusión para poder garantizar la validez interna de los resultados, buscamos investigaciones que incluyeran grupos control y seguimientos. De toda la bibliografía consultada solo 10 investigaciones cumplían con este criterio pudiendo ofrecer las conclusiones de sus estudios datos que garantizaran nuestro objetivo.

Las 10 investigaciones demuestran cambios positivos en variables psicológicas tras pasar por el programa de intervención en mindfulness. Los síntomas psicológicos más evaluados en estos estudios son la ansiedad, depresión y estrés percibido. Los instrumentos utilizados para cada uno el STAI, CES-D y el PSS ${ }^{(20,21,23,24,27)}$.

Los estudios también informan de mejoría en variables físicas. Los síntomas físicos más evaluados en estos estudios son la fatiga seguidos del sueño. Los instrumentos más utilizados para medir la fatiga son BCPT, FACT-B y FSI ${ }^{(20,24,29)}$ y en sueño son el ESPM, Diario de sueño, PSQI y el Acelerómetro ${ }^{(20,26,28)}$. Para la fatiga se informa de mejoría significativa tras la intervención y en los seguimientos, quedando un poco en duda la mejoría en la calidad del sueño tras los seguimientos.

No podemos afirmar que sean intervenciones eficaces para mejorar los síntomas cognitivos e inmunológicos, ya que las dos investigaciones de nuestra revisión que evalúan estos marcadores biológicos son pioneras y no muestran datos 
concluyentes ${ }^{(25,27)}$. Seguir investigando estas variables con diseños rigurosos y poblaciones más amplias en el futuro quizás pueda aportarnos datos reveladores de como la práctica en mindfulness puede ayudar a reducir los síntomas cognitivos que presentan estas pacientes tras los tratamientos y la regulación de su sistema inmunológico.

Tras esto podemos concluir que la intervención con un programa de mindfulness en pacientes supervivientes de cáncer de mama se muestra eficaz para reducir variables psicológicas como; los síntomas de ansiedad, depresión y estrés percibido y variables físicas como la fatiga, mostrando la totalidad de los estudios valores en torno al $(\mathrm{p}=<0,01)$, pudiendo esto mejorar la calidad de vida de estas pacientes.

Tras esto se nos plantea un problema metodológico con respecto a la intervención en mindfulness, ya que en estos estudios hemos encontrado 4 programas diferentes: MBSR, MBSR-BC, MBCR y MAPs. Tras analizarlos hemos observado que todas parten del original programa $\mathrm{MBSR}^{(16)}$ y que en líneas generales están adaptadas a la población de mujeres supervivientes de cáncer de mama en su estructuración; contenido y duración de las sesiones. Con respecto al contenido todas estas modalidades incorporan herramientas para facilitar el afrontamiento de problemas comunes de la enfermedad en estas pacientes como el miedo a la recaída. Con respecto a la duración de las sesiones, mayormente coinciden en desarrollar las sesiones en 2 horas.

Otra pregunta que se plantea es que número de pacientes supervivientes de cáncer de mama es el ideal para intervenir en mindfulness por grupo. Solo 3 artículos ${ }^{(21,28,29)}$ informan en el apartado del procedimiento como distribuyeron en los grupos de intervención a las pacientes. Zhang et al. ${ }^{(21)}$ describe el contenido y objetivo de cada una de las 8 sesiones del programa que aplica de MBSR. Distribuye a las 30 pacientes que inician el programa MBSR y conforman el grupo experimental en 6 grupos con 4 - 6 participantes. Andersen et al. ${ }^{(28)}$ informan que crearon 10 grupos con las 168 participantes del grupo experimental, donde había un mínimo de 10 pacientes y un máximo de 30 . Hoffman et al. ${ }^{(29)}$ en su investigación establecen 12 grupos con un mínimo de 12 y un máximo de 20 practicantes. No hemos encontrado ninguna referencia acerca de la idoneidad del número de participantes en grupos de Mindfulness. Kabat-Zinn et al. ${ }^{(16)}$ en su último libro sobre Mindfulness donde aborda el origen, su significado y aplicaciones no menciona nada a este respecto. Sería esta una cuestión necesaria de estudiar en futuras investigaciones.

Con respecto a los grupos controles vemos que en número de participantes están equiparados a los grupos experimentales. Sería interesante poder comparar en el futuro con diseños rigurosos la intervención en mindfulness con otro tipo de intervenciones psicológicas.

Los seguimientos mayormente en todos estos estudios se realizan a los tres meses, en el futuro sería interesante poder hacerlos extensivos al año de haber finalizado la intervención del programa.

La presente revisión aporta datos a las revisiones anteriores como la de Huang et al. ${ }^{(33)}$ y Rush et al. ${ }^{(34)}$, sobre la eficacia de las intervenciones con mindfulness por el tipo de diseño estudiado en un total de 1.839 pacientes supervivientes de cáncer de mama. A la vez puede servir como guía sobre la modalidad de programa más eficaz a seguir cuando se interviene con mindfulness en pacientes supervivientes de cáncer de mama con respecto a la estructuración y contenido del programa. 


\section{Conclusión}

La presente revisión muestra que las intervenciones psicológicas en mindfulness resultan eficaces en la reducción de sintomatología psicológica (ansiedad, depresión, estrés percibido) y física (fatiga) en pacientes supervivientes con cáncer de mama. Mostrando así su utilidad para ayudar a mejorar la calidad de vida de estas pacientes. No obstante, resultaría interesante seguir estudiando con diseños de investigación rigurosos si también podrían resultar eficaces para reducir síntomas físicos como insomnio, síntomas cognitivos, y mejorar la actividad del sistema inmunológico.

Tras el análisis de los programas de Mindfulness empleados en las expuestas no esta determinado cual es el protocolo de entrenamiento en Mindfulness más idóneo para las supervivientes con cáncer de mama, por lo cual siguen siendo necesarios estudios que vayan encaminados a validar el contenido y estructuración del programa de entrenamiento.

\section{Referencias bibliográficas}

1. REDECAN. Red Española de Registros de Cáncer. [Acceso el 19 de enero de 2017]. Disponible en: http://redecan.org/es/index.cfm

2. EUROCARE-5. Survival of cancer patients in Europe [Acceso 22 octubre 2017]. Disponible en: http://www.eurocare.it/

3. Ferlay J, Soerjomataram I, Dikshit R, Eser S, Mathers C, Rebelo M, et al. Cancer incidence and mortality worldwide: Sources, methods and major patterns in GLOBOCAN. Int J Cancer 2015; 136:E359-86 2012. doi: 10.1002/ijc.29210.

4. Malvezzi M, Bertuccio P, Levi F, La Vecchia C, Negri E. European cancer mortality predictions for the year 2014. Ann Oncol 2014; 25:1650-6. doi: 10.1093/annonc/mdu138

5. Fiszer C, Dolbeault S, Sultan S, Brédart A. Prevalence, intensity, and predictors of the supportive care needs of women diagnosed with breast cancer: a systematic review. Psychooncology 2014;23: 361-74. doi: 10.1002/pon.3432

6. Holland JC, Breitbart WS, Jacobsen Matthew J, Loscalzo JM, McCorkle R. et al. PsychoOncology: New York: Oxford University Press, 2015.

7. Krebber A, Buffart LM, Kleijn G, Riepma IC, de Bree R, Leemans CR, et al. Prevalence of depression in cancer patients: a meta-analysis of diagnostic interviews and self-report instruments. Psychooncology 2014;23:121-30. doi: 10.1002/pon.3409.

8. Tatrow K, Montgomery GH. Cognitive behavioral therapy techniques for distress and pain in breast cancer patients: A meta-analysis. J Behav Med 2006; 29: 17-27.

9. Golant M, Altman T, Martin C. Managing cancer side effects to improve quality of life: a cancer psychoeducation program. Cancer Nurs 2003; 26: 37-44.

10. Fors EA, Bertheussen GF, Thune I, Juvet LK, Elvsaas IKØ, Oldervoll L., et al. Psychosocial interventions as part of breast cancer rehabilitation programs? Results from a systematic review. Psychooncology 2011; 20:909-18. doi: 10.1002/pon.1844.

11. Stagl, J. M., Bouchard, L. C., Lechner, S. C., Blomberg, B. B., Gudenkauf, L. M., Jutagir et al. Long-term psychological benefits of cognitive-behavioral stress management for women with breast cancer: 11-year follow-up of a randomized controlled trial. Cancer 2015; 121: 1873-81. 
12. Jassim GA, Whitford DL, Grey I. M. Psychological interventions for women with nonmetastatic breast cancer. Cochrane Database Syst Rev 2010; 28;(5):CD008729. doi: 10.1002/14651858.CD008729.pub2.

13. Vallejo MA, Ortega J. La utilización del mindfulness en el paciente oncológico. Psicooncología 2008; 4: 35-41.

14. Palao Á, Rodríguez B, PriedeA, Maeso A, Arranz H. Cambios psicológicos e intervenciones basadas en mindfulness para los supervivientes de un cáncer. Psicooncología 2011; 8: 7-20. doi: 10.5209/rev_PSIC.2011.v8.n1.1

15. Shennan C, Payne S, Fenlon D. What is the evidence for the use of mindfulness-based interventions in cancer care? A review. Psychooncology 2011; 20: 681-97. doi: 10.1002/ pon.1819.

16. Kabat-Zinn J, Williams MG. Mindfulness: Su origen, significado y aplicaciones: Barcelona: Kairós, 2017.

17. Segal Z, Williams M, Teasdale J. Terapia cognitiva basada en el mindfulness para la depresión. Kairós, 2015.

18. Carlson LE. Mindfulness-based interventions for coping with cancer. Ann N Y Acad Sc 2016; 1373: 5-12. doi: 10.1111/nyas.13029.

19. Bartley T. Terapia cognitiva basada en el mindfulness para el cáncer. Bilbao: Desclee de Brouwer, 2013.

20. Reich RR, Lengacher CA, Alinat CB, Kip KE, Paterson C, Ramesar S, et al. Mindfulnessbased stress reduction in post-treatment breast cancer patients: immediate and sustained effects across multiple symptom clusters. J Pain Symptom Manage 2017; 53: 85-95. doi: 10.1016/j.jpainsymman.2016.08.005.

21. Zhang JY, Zhou YQ, Feng ZW, Fan YN, Zeng GC, Wei L. Randomized controlled trial of mindfulness-based stress reduction (MBSR) on posttraumatic growth of Chinese breast cancer survivors. Psychology Health Med 2017; 22: 94-109. Doi. 10.1080/13548506.2016.1146405

22. Carlson LE, Tamagawa R, Stephen J, Drysdale E, Zhong L, Speca M. Randomizedcontrolled trial of mindfulness-based cancer recovery versus supportive expressive group therapy among distressed breast cancer survivors (MINDSET): long-term follow-up results. Psychooncology 2016; 25: 750-9. doi: 10.1002/pon.4150.

23. Lengacher CA, Reich RR, Paterson CL, Ramesar S, Park JY, Alinat C, et al. Examination of broad symptom improvement resulting from mindfulness-based stress reduction in breast cancer survivors: a randomized controlled trial. J Clin Oncol 2016; 34: 2827-34. doi: 10.1200/JCO.2015.65.7874.

24. Bower JE, Crosswell AD, Stanton AL, Crespi CM, Winston D, Arevalo J, et al. Mindfulness meditation for younger breast cancer survivors: A randomized controlled trial. Cancer 2015; 121:1231-40. doi: 10.1002/cncr.29194.

25. Lengacher CA, Reich RR, Kip KE, Paterson CL, Park HY, Ramesar S, et al. Moderating effects of genetic polymorphisms on improvements in cognitive impairment in breast cancer survivors participating in a 6-week mindfulness-based stress reduction program. Biological research for nursing. 2015; 17: 393-404. doi: 10.1177/1099800415577633

26. Lengacher CA, Reich RR, Paterson CL, Jim HS, Ramesar S, Alinat CB, et al. The effects of mindfulness-based stress reduction on objective and subjective sleep parameters in women with breast cancer: a randomized controlled trial. Psychooncology 2015; 24: 42432. doi: 10.1002/pon.3603 
27. Lengacher CA, Reich RR, Kip KE, Barta M, Ramesar S, Paterson CL, et al. Influence of mindfulness-based stress reduction (MBSR) on telomerase activity in women with breast cancer (BC). Biol Res Nurs 2014; 16: 438-47. doi: 10.1177/1099800413519495

28 Andersen SR, Würtzen H, Steding-Jessen M, Christensen J, Andersen KK, Flyger $\mathrm{H}$, et al. Effect of mindfulness-based stress reduction on sleep quality: results of a randomized trial among Danish breast cancer patients. Acta Oncol 2013; 52: 336-44. doi: 10.3109/0284186X.2012.745948

29. Hoffman CJ, Ersser SJ, Hopkinson JB, Nicholls PG, Harrington JE, Thomas PW. Effectiveness of mindfulness-based stress reduction in mood, breast-and endocrinerelated quality of life, and well-being in stage 0 to III breast cancer: A randomized, controlled trial. J Clin Oncol 2012; 30: 1335-42. doi: 10.1200/JCO.2010.34.0331.

30. Mindful Awareness Research Center (MARC) [Acceso 10 enero 2018]. Disponible en: http://marc.ucla.edu/

31. Cramer H, Lauche R, Paul A, Dobos G. Mindfulness-based stress reduction for breast cancer- a systematic review and meta-analysis. Curr Oncol 2012; 19: 343-52. doi: 10.3747/co.19.1016.

32. Zainal NZ, Booth S, Huppert FA. The efficacy of mindfulness-based stress reduction on mental health of breast cancer patients: a meta-analysis. Psychooncology 2013; 22: 145765. doi: 10.1002/pon.3171

33. Huang HP, He M, Wang HY, Zhou M. A meta-analysis of the benefits of mindfulnessbased stress reduction (MBSR) on psychological function among breast cancer (BC) survivors. Breast Cancer 2016; 23: 568-76. doi: 10.1007/s12282-015-0604-0

34. Rush SE, Sharma M. Mindfulness-Based Stress Reduction as a Stress Management Intervention for Cancer Care: A Systematic Review. J Evid Based Complementary Altern Med 2017; 22: 348-60. doi: 10.1177/2156587216661467 